\title{
Time Dependent Changes in Ag Doped YBCO Superconductors
}

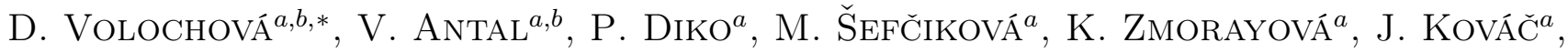 \\ H.W. WeBER ${ }^{b}$ AND X. CHAUD ${ }^{c}$ \\ ${ }^{a}$ Materials Physics Laboratory, Institute of Experimental Physics, Slovak Academy of Sciences \\ Watsonova 47, 04001 Košice, Slovak Republic \\ ${ }^{b}$ Vienna University of Technology, Atominstitut, Stadionallee 2, 1020 Vienna, Austria \\ ${ }^{c}$ CNRS/CRETA, 25, Avenue des Martyrs, 38042 Grenoble Cedex 9, France
}

\begin{abstract}
Magnetization measurements at $77 \mathrm{~K}$ were conducted on the single-crystalline samples of YBCO superconductor doped with Ag prepared by the top-seeded melt-growth process. The single-grain samples had a nominal composition $\mathrm{Y}_{1.5} \mathrm{Ba}_{2}(\mathrm{Cu}, \mathrm{Ag})_{3} \mathrm{O}_{y}$. Magnetization measurements on the samples, oxygenated by two different processes: by standard oxygenation and by high pressure oxygenation, repeated after 24 months, revealed time dependent changes in magnetization behaviour and critical transition temperatures, $T_{\mathrm{c}}$, which could be associated with redistribution of oxygen vacancies and their interaction with $\mathrm{Ag}$ dopant atoms.

PACS numbers: 74.25.Ha, 74.25.Sv, 74.62.Bf, 74.62.Dh, 74.62.Fj
\end{abstract}

\section{Introduction}

Melt-processed (RE)-Ba-Cu-O (REBCO, RE $=\mathrm{Y}$, $\mathrm{Gd}, \mathrm{Nd}$ etc.) bulk superconductors represent a class of materials with great superconducting and magnetic properties which make them very attractive for wide-ranging technical applications. Nevertheless, one of the main interests is still focused to achieve higher critical temperatures, $T_{\mathrm{c}}$, and further improvement of critical current density, $J_{c}$, especially at higher magnetic fields. It is possible to considerably enhance critical current density, $J_{\mathrm{c}}$, of these materials for example by irradiation [1] or chemical doping [2]. In the case of YBCO melt-solidified bulks it was already shown that $\mathrm{Ag}$ addition can enhance pinning properties [3] besides the improvement of mechanical properties [4]. Additionally, Feng et al. consider that Ag-doping of YBCO can effectively prevent samples from degradation [5] which is another important question for practical applications of these materials. We report on time dependent changes in the pinning behaviour and critical transition temperatures, $T_{\mathrm{c}}$, of Ag-doped YBCO bulk superconductors. The influence of two different thermo-chemical treatments is shown.

\section{Experimental details}

Ag-doped YBCO bulk single-grain superconductors with a composition: $\mathrm{Y}_{1.5} \mathrm{Ba}_{2}\left(\mathrm{Cu}_{1-x} \mathrm{Ag}_{x}\right)_{3} \mathrm{O}_{y}(x=0.0025$ to 0.05$)+25 \mathrm{~mol} . \% \mathrm{Y}_{2} \mathrm{O}_{3}+1 \mathrm{wt} \% \mathrm{CeO}_{2}$ were fabricated by the top-seeded melt-growth process (TSMG) in air atmosphere. $\mathrm{SmBa}_{2} \mathrm{Cu}_{3} \mathrm{O}_{y}$ single crystals were used as nucleation seeds. Samples for oxygenation and magnetization measurements had a shape of a slab with the

\footnotetext{
* corresponding author; e-mail: volochova@saske.sk
}

dimensions $2 \times 2 \times 0.5 \mathrm{~mm}^{3}$, the smallest dimension was parallel to the $c$-axis.

The samples were oxygenated by two different processes: standard oxygenation (SO) and high pressure oxygenation (HPO). During SO the samples were heated to $800^{\circ} \mathrm{C}$ in a flowing oxygen and kept there for $2 \mathrm{~h}$, then cooled down to $400^{\circ} \mathrm{C}$ and held at this temperature for $240 \mathrm{~h}$ and finally cooled down to room temperature. During HPO the samples were oxygenated under an oxygen pressure of 160 bars at $750{ }^{\circ} \mathrm{C}$ for $24 \mathrm{~h}$ with progressively increasing oxygen pressure.

The magnetization measurements were done by a vibrating-sample magnetometer (VSM) with magnetic fields up to $6 \mathrm{~T}$ applied parallel to the $c$-axis of the crystal, at the temperature of $77 \mathrm{~K}$. The critical current densities, $J_{\mathrm{c}}$, were calculated from the magnetization hysteresis loops using the extended Bean model [6] for rectangular samples. The critical transition temperatures, $T_{\mathrm{c}}$, and the transition widths, $\Delta T_{\mathrm{c}}$, were determined from the magnetic transition curves taken after zero-field cooling as the $T_{\mathrm{c}}(50 \%)$ and, as the $T_{\mathrm{c}}(90 \%)-T_{\mathrm{c}}(10 \%)$, respectively, of this curve in an applied external magnetic field of $2 \mathrm{mT}$.

\section{Results and discussion}

The critical transition temperatures, $T_{\mathrm{c}}$, and transition widths, $\Delta T_{\mathrm{c}}$, of $\mathrm{YBa}_{2}\left(\mathrm{Cu}_{1-x} \mathrm{Ag}_{x}\right)_{3} \mathrm{O}_{7-\delta}$ samples measured in short time after oxygenation and repeated after 24 months are presented in Fig. 1a and b, respectively. The diagrams show that even small changes in the composition of YBCO crystal can influence $T_{\mathrm{c}}$ and $\Delta T_{\mathrm{c}}$ for both SO and HPO. It can be seen, especially, in the case of the $\mathrm{YBa}_{2}\left(\mathrm{Cu}_{0.95} \mathrm{Ag}_{0.05}\right)_{3} \mathrm{O}_{7-\delta}$ sample. The decrease of $T_{\mathrm{c}}$ and increase of $\Delta T_{\mathrm{c}}$ for this concentration of $\mathrm{Ag}$ 
dopant can suggest that the $\mathrm{Cu}$ atoms in the YBCO lattice are partially substituted by the Ag atoms.

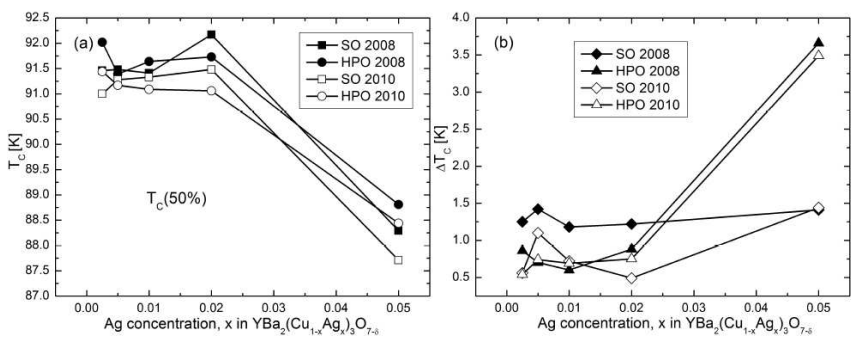

Fig. 1. Critical transition temperatures, $T_{\mathrm{c}}$ (a), and transitions widths, $\Delta T_{\mathrm{c}}(\mathrm{b})$, as a function of $\mathrm{Ag}$ concentration, $x$.

After 24 months, the decrease of $T_{\mathrm{c}}$ was observed for all samples after $\mathrm{SO}$ as well as for samples after HPO (Fig. 1a). No significant changes were found in the dependences $\Delta T_{\mathrm{c}}(x)$ for samples after HPO (Fig. 1b) whereas the decrease of $\Delta T_{\mathrm{c}}$ was found for almost all samples after SO. This can be related to higher non-homogeneous distribution of oxygen in entire sample after SO in comparison to samples after HPO. We suppose that samples after SO become more homogeneous after aging.

Figure 2 shows the dependences of the critical current density, $J_{\mathrm{c}}$, on applied magnetic field, $B$, at $77 \mathrm{~K}$ for five different $\mathrm{Ag}$ concentrations, $x$ in $\mathrm{YBa}_{2}\left(\mathrm{Cu}_{1-x} \mathrm{Ag}_{x}\right)_{3} \mathrm{O}_{7-\delta}$ oxygenated by SO (Fig. 2a) and HPO (Fig. 2b) measured in 2008. Magnetization measurements on the same samples, repeated after 24 months, are shown in Fig. 2c and $\mathrm{d}$, respectively.
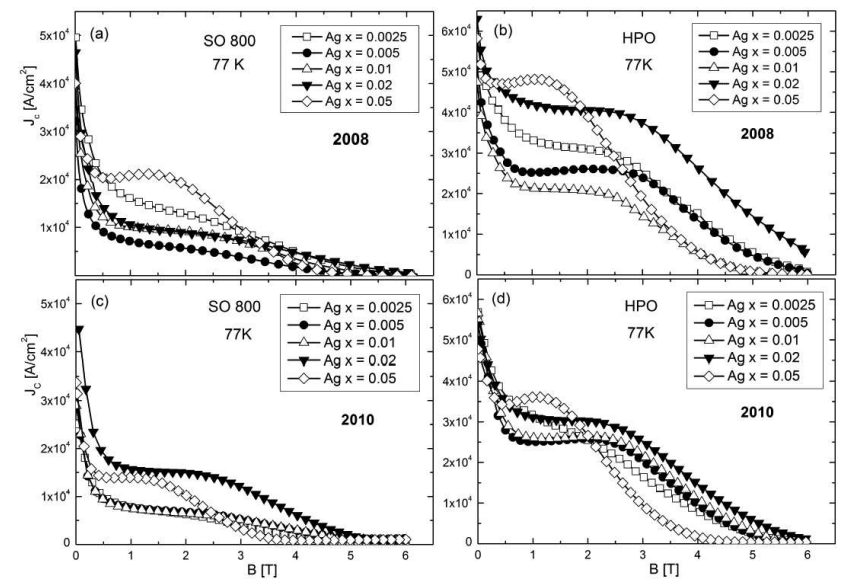

Fig. 2. Field dependences of critical current density, $J_{\mathrm{c}}$, at $77 \mathrm{~K}$ for all $\mathrm{Ag}$ concentrations, $x$ in $\mathrm{YBa}_{2}\left(\mathrm{Cu}_{1-x} \mathrm{Ag}_{x}\right)_{3} \mathrm{O}_{7-\delta}$ oxygenated by $\mathrm{SO}$ (a) and HPO (b) repeated after 24 months (c) for SO and (d) for HPO.

In the case of SO treatment (Fig. 2a), the peak effect was observed only at the highest nominal dopant concentration $x=0.05$. The explanation of this behaviour was given by detailed observation [7] which revealed in- homogeneous distribution of $\mathrm{Ag}$ in the single-grain bulk samples caused by the low Ag partition coefficient at solidification. Significant improvement of pinning properties of Ag-doped YBCO bulk superconductors can be reached by HPO [8] (Fig. 2b) which eliminates the formation of oxygenation cracks and increases the effective cross-section of the samples [9].

Magnetization measurements repeated after 24 months show changes in the field dependence of the critical current densities, $J_{\mathrm{c}}$ (Fig. 2c,d). The decrease of $J_{\mathrm{c}}$ for all concentrations of the $\mathrm{Ag}$ dopant was observed, except of the sample after HPO with concentration of $\mathrm{Ag} x=0.01$ and $x=0.02$ for the sample after SO, where the increase of $J_{c}$ was found. Possible origin of such changes is a redistribution of pinning centres in the samples caused by diffusion of oxygen vacancies and their interaction with Ag dopant atoms.

\section{Acknowledgments}

This work was realized within the frame of the projects "Centre of Excellence of Advanced Materials with Nano- and Submicron Structure" (ITMS 262200120019), New Materials and Technologies for Energetics (ITMS 26220220061), Research and Development of Second Generation YBCO Bulk Superconductors (ITMS 26220220041), which are supported by the Operational Program "Research and Development" financed through European Regional Development Fund by VEGA project No. 2/0211/10, project ERANET-ESO and by the Centre of Excellence of Slovak Academy of Sciences NANOSMART.

\section{References}

[1] R. Gonzalez-Arrabal, M. Eisterer, H.W. Weber, G. Fuchs, P. Verges, G. Krabbes, Appl. Phys. Lett. 81, 8682 (2002).

[2] L. Shlyk, G. Krabbes, P. Diko, K. Nenkov, G. Fuchs, Inst. Phys. Conf. Ser. 181, 1401 (2004).

[3] T. Nakashima, Y. Ishii, T. Katayama, H. Ogino, S. Horii, J. Shimoyama, K. Kishio, J. Phys. Conf. Ser. 97, 012007 (2008).

[4] P. Diko, Supercond. Sci. Technol. 17, R45 (2004).

[5] Q. Feng, X. Zhu, S. Feng, H. Zhang, Z. Gan, Supercond. Sci. Technol. 6, 715 (1993).

[6] C.P. Bean, Phys. Rev. Lett. 8, 250 (1962).

[7] P. Diko, V. Antal, M. Kaňuchová, M. Jirsa, K. Jurek, Physica C 470, 155 (2010).

[8] P. Diko, X. Chaud, V. Antal, M. Kaňuchová, M. Šefčiková, J. Kováč, Supercond. Sci. Technol. 21, 115008 (2008).

[9] M. Eisterer, S. Haindl, M. Zehetmayer, R. Gonzalez-Arraball, H.W. Weber, D. Litzkendorf, M. Zeisberger, T. Habisreuther, W. Gawalek, L. Shlyk, G. Krabbes, Supercond. Sci. Technol. 19, S530 (2006). 